\title{
Seroprevalencia de infección por dengue y factores asociados en residentes del centro poblado Fila Alta
}

\author{
Seroprevalence of dengue infection and associated factors in residents of Fila Alta \\ village
}

Aydee Facundo 1,a, Gabriela Sánchez 1,a, Christian Rivera-Salazar 1,b , Cinthya Santa Cruz-López « 1,c

\author{
Filiación y grado académico \\ 1 Universidad Nacional de Jaén, Cajamarca, \\ Perú. \\ a Bachiller en Tecnología Médica. \\ ${ }^{b}$ Maestro en Ciencias. \\ Doctora en Ciencias Biomédicas.
}

(iD) ORCID iD de Aydee Facundo https://orcid.org/0000-0002-2469-2051

(iD) ORCID iD de Gabriela Sánchez https://orcid.org/0000-0002-9081-0036

(iD) ORCID iD de Christian Rivera-Salazar https://orcid.org/0000-0002-4959-2188

(iD) ORCID iD de Cinthya Santa Cruz-López https://orcid.org/0000-0002-7352-058X

\section{Contribución de autoría}

AF y GS: concepción y diseño del estudio, búsqueda bibliográfica, recolección de los datos y participación en la elaboración del manuscrito.

CRS: búsqueda bibliográfica, recolección de los datos, análisis estadístico de los datos y participación en la elaboración del manuscrito.

CSCL: búsqueda bibliográfica, participación en la elaboración y revisión del borrador y de la versión final del manuscrito.

Fuentes de financiamiento

El trabajo de investigación fue financiado con recursos propios de los investigadores.

Conflictos de interés

Los autores declaran no tener conflictos de interés.

Recibido: 08-02-2021

Arbitrado por pares

Aceptado: 15-04-2021

Citar como

Facundo A, Sánchez G, Rivera-Salazar C, Santa Cruz-López C. Seroprevalencia de infección por dengue y factores asociados en residentes del centro poblado Fila Alta. Rev Peru Cienc Salud. 2021; 3(2): 104-9. doi: https://doi.org/10.37711/rpcs.2021.3.2.312

\section{Correspondencia}

Cinthya Yanina Santa Cruz-López

Dirección: Avenida Luis Gonzales 1342.

Chiclayo, Perú.

CP.: 14001

Celular: 945391136

Email: cisantacruzl@gmail.com

\section{RESUMEN}

Objetivo. Determinar la seroprevalencia de infección por dengue e identificar los factores asociados en los residentes del centro poblado Fila Alta, distrito de Jaén, departamento de Cajamarca, durante los meses de septiembre a diciembre del 2019. Métodos. Estudio observacional, transversal, prospectivo con diseño no experimental de enfoque descriptivocorrelacional. La muestra estuvo conformada por 172 pobladores de ambos géneros y el muestreo fue aleatorio simple. La seroprevalencia de anticuerpos IgG se determinó mediante la prueba inmunoenzimática indirecta (ELISA) y se aplicó una encuesta estructurada para identificar los factores asociados al dengue. Asimismo, se utilizó la prueba de chi cuadrado para establecer la relación entre las variables estudiadas. Resultados. El 19,2\% de pobladores presentaron anticuerpos IgG frente al dengue, siendo más frecuente en las mujeres (12,2\%). El 9,3\% (OR: 0,18; RP: 0,16; IC95\%: 0,09-0,23) de estudiantes y el 5,8\% (OR: 0,31 RP: 0,24; IC95 \%: 0,11-0,37) de amas de casa resultaron seropositivos al dengue. Además, el 15,7\% (OR: 0,28; RP: 0,22; IC95 \%: 0,15$0,29)$ de pobladores seropositivos se abastecía de agua a través de tanques y el material más utilizado en la construcción de sus viviendas fue el adobe (8,7 \%; OR: 0,94; RP: 0,48; IC95 \%: 0,30-0,66). Conclusiones. La seroprevalencia de la infección por dengue es alta y está relacionada significativamente con la ocupación de los pobladores y el material de las paredes de sus viviendas.

Palabras clave: dengue; estudios seroepidemiológicos; ensayo de inmunoadsorción enzimática; factores predisponentes (Fuente: DeCS-BIREME).

\section{ABSTRACT}

Objective. The objectives of the study were to determine the seroprevalence of dengue infection and to identify the associated factors in the residents of the Fila Alta village, district of Jaén, department of Cajamarca, during the months of September to December 2019. Methods. Observational, cross-sectional, prospective study with a non-experimental design with a descriptive-correlational approach. The sample consisted of 172 inhabitants of both genders and the sampling was simple random. The seroprevalence of IgG antibodies was determined by indirect enzyme-linked immunosorbent assay (ELISA) and a structured survey was applied to identify the factors associated with dengue. Likewise, the chi-square test was used to establish the relationship between the variables studied. Results. IgG antibodies to dengue fever were present in $19.2 \%$ of the population, being more frequent in women (12.2\%). Dengue seropositivity was found in $9.3 \%$ (OR: 0.18 ; PR: 0.16 ; $95 \% \mathrm{Cl}: 0.09-0.23$ ) of students and $5.8 \%$ (OR: 0.31 PR: $0.24 ; 95 \% \mathrm{Cl}: 0.11-$ 0.37 ) of housewives. In addition, $15.7 \%$ (OR: 0.28 ; PR: $0.22 ; 95 \% \mathrm{Cl}: 0.15-0.29$ ) of seropositive villagers obtained their water supply from tanks and the most common material used in the construction of their houses was adobe (8.7 \%; OR: 0.94; PR: $0.48 ; 95 \% \mathrm{Cl}: 0.30-0.66)$. Conclusions. The seroprevalence of dengue infection is high and is significantly related to the occupation of the inhabitants and the material of the walls of their houses.

Keywords: dengue; seroepidemiological studies; enzyme-linked immunosorbent assay; predisposing factors (Source: MeSH - NLM). 


\section{INTRODUCCIÓN}

El dengue es una enfermedad viral transmitida mediante la picadura de un mosquito del género Aedes, principalmente el Aedes aegypti ${ }^{(1)}$. El mosquito hembra infectado es capaz de transmitir al virus del dengue (DENV) a lo largo de toda su vida ${ }^{(2)}$. La infección por dengue presenta un cuadro clínico muy variable, generalmente con una rápida recuperación del paciente; sin embargo, puede progresar a formas graves e incluso mortales. De modo que la enfermedad se manifiesta como dengue con signos de alarma y dengue grave ${ }^{(3)}$.

Se estima que cada año se producen entre 50 y 100 millones de nuevas infecciones por dengue en más de 100 países endémicos y alrededor de 20000 muertes anuales por dengue grave ${ }^{(4)}$. Durante el año 2017, en el Perú se reportaron 76093 casos de dengue, de los cuales el $11 \%$ fueron casos de dengue con signos de alarma y $0,3 \%$ de dengue grave ${ }^{(5)}$.

La enfermedad ocasionada por el virus del dengue genera elevados costos para el estado peruano y la población en general, debido a que disminuye la productividad laboral y promueve el ausentismo escolar, a consecuencia del tiempo necesario para la recuperación del paciente e incluso el costo de hospitalización, sobre todo en los casos de dengue grave ${ }^{(6)}$. Esta arbovirosis es cada vez más frecuente en zonas como la costa norte y la Amazonía peruana. Su elevada frecuencia está relacionada con las altas temperaturas, así como los largos periodos de lluvias y humedad presentes en diferentes zonas del país que favorecen la continuidad del ciclo biológico del vector ${ }^{(4)}$.

Otros factores que influyen en la diseminación del $A$. aegypti, son el inadecuado almacenamiento y eliminación de los recipientes de agua, escasa disponibilidad de agua para el consumo, eliminación de residuos sólidos, ineficientes medidas de control vectorial, desconocimientos de los síntomas del dengue, incremento de la migración poblacional, entre otros ${ }^{(7,8)}$. Es así que una persona infectada que se desplaza de un lugar a otro puede fácilmente diseminar el virus, debido a que el vector se encuentra ampliamente distribuido en el país ${ }^{(9)}$.

El diagnóstico de laboratorio se basa principalmente en el aislamiento del patógeno y la utilización de pruebas serológicas, siendo estas últimas las más empleadas por su alto grado de sensibilidad, especificidad, bajo costo y reproducibilidad de los resultados obtenidos ${ }^{(10)}$. Una de las técnicas más utilizadas es el ensayo inmunoenzimático (ELISA), el cual permite detectar de forma cualitativa la presencia de inmunoglobulinas $\mathrm{M}$ y $\mathrm{G}$ frente al dengue.
Por lo que es de gran importancia diagnosticar oportunamente y hacer seguimiento a los casos de dengue en el Perú, evitando posibles complicaciones y nuevas epidemias, ya que las condiciones climáticas, sanitarias y económicas presentes en distintas zonas del país incrementan el riesgo de infección por este patógeno. Ante ello, se planteó como objetivos determinar la seroprevalencia de infección por dengue e identificar los factores asociados en los residentes del centro poblado Fila Alta, distrito de Jaén, departamento de Cajamarca, durante los meses de septiembre a diciembre del 2019.

\section{MÉTODOS}

\section{Tipo de estudio}

El estudio realizado fue de tipo observacional, transversal y prospectivo y se utilizó un diseño no experimental con enfoque Descriptivo-correlacional.

\section{Población y muestra}

La población de estudio estuvo constituida por 1200 habitantes del centro poblado Fila Alta, distrito de Jaén, departamento de Cajamarca (Latitud: -5.737449 , Longitud: -78.789090 ) a 814 m s.n.m., los cuales fueron evaluados durante el periodo de septiembre a diciembre del año 2019. Por su parte, la muestra estuvo conformada por 172 pobladores de ambos géneros con edades comprendidas entre 10 y 50 años. El tamaño de la muestra se calculó con la fórmula estadística para poblaciones conocidas y se aplicó un muestreo de tipo probabilístico-aleatorio simple.

\section{Instrumentos de recolección de datos}

La información acerca de los factores asociados al dengue se recopiló utilizando como técnica de recolección una encuesta dirigida a los residentes del centro poblado Fila Alta en el distrito de Jaén. En dicha encuesta fueron incluidas preguntas acerca del género, edad, ocupación, material utilizado para la construcción de las viviendas y abastecimiento de agua. El instrumento de recolección de datos fue un cuestionario estructurado y validado por Niño y Yong ${ }^{(11)}$.

\section{Procedimientos de la recolección de datos}

Para determinar la infección por dengue, se utilizó la prueba inmunoenzimática indirecta para la detección de anticuerpos $\lg G$ Capture ELISA ${ }^{\oplus}$ en suero sanguíneo (VIRCELL), de acuerdo a lo establecido por el fabricante ${ }^{(12)}$.

\section{Aspectos éticos}

El estudio fue revisado y aprobado por la Comisión Central de Investigación de la Universidad Nacional de Jaén. Los pobladores accedieron a participar voluntariamente en el estudio, a través de la firma de un consentimiento 
informado. Además, las muestras de sangre y los cuestionarios recopilados fueron identificados solo con codificación numérica seriada, asegurando la utilización de la información obtenida solo con fines de investigación, siguiendo lo establecido en la Declaración de Helsinki.

\section{Análisis de datos}

El análisis descriptivo de los datos se realizó con el paquete estadístico Minitab ${ }^{\circledR} 19$ para Windows ${ }^{\circledast}$ versión 8 . Se calcularon las frecuencias absolutas y relativas para variables cualitativas, así como las y medias para las variables cuantitativas. Además, se utilizó la prueba de chi cuadrado para determinar la relación entre la seroprevalencia de infección por dengue y los factores asociados en los pobladores de Fila Alta.

\section{RESULTADOS}

Se evaluó el suero sanguíneo de 172 pobladores de ambos géneros, pertenecientes al centro poblado Fila Alta. El 19,2 \% resultó seropositivo a la infección por dengue, perteneciendo el $12,2 \%$ al género femenino y el $6,7 \%$ al género masculino (ver tabla 1). No se estableció relación significativa entre la seroprevalencia y el género de los pobladores $(p>0,05)$.

En la tabla 2 se reportó que el $61,6 \%$ de los residentes pertenecía al grupo etario de 10 a 20 años, de los cuales
Tabla 1. Seroprevalencia de infección por dengue según el género de los residentes del centro poblado

\begin{tabular}{|c|c|c|c|c|c|c|c|c|}
\hline \multirow{3}{*}{ Género } & \multicolumn{8}{|c|}{ Seroprevalencia de dengue } \\
\hline & \multicolumn{2}{|c|}{ Seropositivo } & \multicolumn{2}{|c|}{ Seronegativo } & \multicolumn{2}{|c|}{ Total } & \multirow{2}{*}{$x^{2}$} & \multirow{2}{*}{ p } \\
\hline & fi & $\%$ & fi & $\%$ & fi & $\%$ & & \\
\hline Femenino & 21 & 12,2 & 86 & 50,0 & 107 & 62,2 & & \\
\hline Masculino & 12 & 6,7 & 53 & 30,8 & 65 & 37,8 & 0,035 & 0,851 \\
\hline Total & 33 & 19,2 & 139 & 80,8 & 172 & 100,0 & & \\
\hline
\end{tabular}

el 8,7 \% (OR: 0,16; RP: 0,14; IC95 \%: 0,08-0,21) presentó anticuerpos lgG anti dengue. El 59,9\% de los pobladores evaluados fueron estudiantes, seguido de un $24,4 \%$ de amas de casa, resultado un 9,3\% (OR: 0,18; RP: 0,16; IC95 \%: 0,09-0,23) y 5,8 \% (OR: 0,31 RP: 0,24; IC95 \%: $0,11-0,37)$ seropositivos al dengue, respectivamente. En relación al abastecimiento de agua, el 15,7 \% (OR: 0,28; RP: 0,22; IC95 \%: 0,15-0,29) de pobladores seropositivos al dengue obtenía el agua a partir de tanques. Además, el material utilizado en las casas de los pobladores con seropositividad al dengue fue principalmente el adobe (8,7\%; OR: 0,94; RP: 0,48; IC95 \%: 0,30-0,66). Al utilizar la prueba de chi cuadrado se estableció relación significativa entre la ocupación y material de construcción con la infección por dengue $(p<0,05)$.

Tabla 2. Factores asociados a la infección por dengue en los residentes del centro poblado

\begin{tabular}{|c|c|c|c|c|c|c|c|c|c|c|c|c|c|}
\hline \multirow{3}{*}{$\begin{array}{l}\text { Factores } \\
\text { asociados }\end{array}$} & \multicolumn{13}{|c|}{ Seroprevalencia de dengue } \\
\hline & & \multicolumn{2}{|c|}{ Seropositivos } & \multicolumn{2}{|c|}{ Seronegativos } & \multicolumn{2}{|c|}{ Total } & \multirow{2}{*}{ OR } & \multirow{2}{*}{$\mathbf{R P}$} & \multicolumn{2}{|c|}{$\begin{array}{l}\text { Intervalo de } \\
\text { confianza (IC) }\end{array}$} & \multirow{2}{*}{$\mathbf{x}^{2}$} & \multirow{2}{*}{$\mathbf{p}$} \\
\hline & & fi & $\%$ & fi & $\%$ & fi & $\%$ & & & Inferior & Superior & & \\
\hline \multirow{4}{*}{ Grupo etario } & $10-20$ & 15 & 8,7 & 91 & 52,9 & 106 & 61,6 & 0,16 & 0,14 & 0,08 & 0,21 & \multirow{4}{*}{6,11} & \multirow{4}{*}{0,11} \\
\hline & $21-30$ & 6 & 3,5 & 19 & 11,1 & 25 & 14,5 & 0,32 & 0,24 & 0,07 & 0,41 & & \\
\hline & $31-40$ & 7 & 4,1 & 12 & 6,9 & 19 & 11,0 & 0,58 & 0,37 & 0,15 & 0,59 & & \\
\hline & $41-50$ & 5 & 2,9 & 17 & 9,9 & 22 & 12,8 & 0,29 & 0,23 & 0,05 & 0,40 & & \\
\hline \multirow{4}{*}{ Ocupación } & Estudiante & 16 & 9,3 & 87 & 50,6 & 103 & 59,9 & 0,18 & 0,16 & 0,09 & 0,23 & \multirow{4}{*}{8,87} & \multirow{4}{*}{$0,03^{*}$} \\
\hline & Ama de casa & 10 & 5,8 & 32 & 18,6 & 42 & 24,4 & 0,31 & 0,24 & 0,11 & 0,37 & & \\
\hline & $\begin{array}{c}\text { Trabajo de } \\
\text { campo }\end{array}$ & 1 & 0,6 & 13 & 7,6 & 14 & 8,1 & 0,08 & 0,07 & 0,00 & 0,21 & & \\
\hline & otros & 6 & 3,5 & 7 & 2,3 & 13 & 2,9 & 0,86 & 0,46 & 0,19 & 0,73 & & \\
\hline \multirow{2}{*}{$\begin{array}{l}\text { Abastecimiento } \\
\text { de agua }\end{array}$} & Tanque de agua & 27 & 15,7 & 97 & 56,4 & 124 & 72,1 & 0,28 & 0,22 & 0,15 & 0,29 & \multirow[b]{2}{*}{1,92} & \multirow[b]{2}{*}{0,17} \\
\hline & $\begin{array}{c}\text { Red } \\
\text { intradomiciliaria }\end{array}$ & 6 & 3,5 & 42 & 24,4 & 48 & 27,9 & 0,14 & 0,13 & 0,03 & 0,22 & & \\
\hline \multirow{3}{*}{$\begin{array}{l}\text { Material de } \\
\text { construcción }\end{array}$} & Adobe & 15 & 8,7 & 16 & 9,3 & 31 & 18,0 & 0,94 & 0,48 & 0,30 & 0,66 & \multirow{3}{*}{21,62} & \multirow{3}{*}{$0,00^{*}$} \\
\hline & Ladrillo & 14 & 8,1 & 80 & 46,5 & 94 & 54,6 & 0,18 & 0,15 & 0,08 & 0,22 & & \\
\hline & Madera & 4 & 2,3 & 43 & 25,0 & 47 & 27,3 & 0,09 & 0,09 & 0,01 & 0,16 & & \\
\hline
\end{tabular}




\section{DISCUSIÓN}

El dengue es endémico en muchas zonas del Perú y los cuatro serotipos del virus (DENV1, DENV2, DENV3 y DENV4) ya han sido reportados dentro del territorio peruano ${ }^{(9)}$. Los casos de dengue más graves (frecuentes en personas que ya padecieron la enfermedad previamente) están asociados a la infección primaria ocasionada por los serotipos DENV-1 y DENV-3, mientras que el desarrollo de un dengue sistémico se relaciona con las infecciones secundarias causadas por el serotipo DENV-2 ${ }^{(13)}$.

La presente investigación determinó la seroprevalencia de infección por dengue y los factores asociados en residentes del centro poblado Fila Alta; una zona cuyo clima tropical y constantes lluvias favorece el desarrollo del $A$. aegypti y predispone a sus habitantes a la infección con el virus del dengue.

En la tabla 1 se reportó que el 19,2 \% de pobladores presentó anticuerpos lgG frente al dengue, siendo más frecuente en las mujeres (12,2\%). Lo resultados obtenidos son similares a los reportados por Neira ${ }^{(14)}$ y Molineros et al. ${ }^{(15)}$, quienes encontraron una seropositividad del $21,4 \%$ en un centro poblado de la ciudad de Trujillo y del 20,3\% en municipios del Valle de Cauca, respectivamente. Dichos investigadores también evidenciaron mayor seroprevalencia de anticuerpos lgG en las mujeres respecto a los varones. Cabe señalar que las mujeres que habitan en el centro poblado Fila Alta generalmente permanecen en sus viviendas, donde realizan labores domésticas, exponiéndose al vector del dengue, cuyo habitad es principalmente intra y peridomiciliario.

El A. aegypti se desarrolla fácilmente dentro de los contenedores sombreados y con agua limpia. Los mosquitos hembra depositan sus huevos en la superficie del líquido y en las paredes de los contenedores. En las zonas con climas muy lluviosos, como la provincia de Jaén, los mosquitos también pueden depositar sus huevos dentro de llantas, latas, botellas u otros recipientes que contengan el agua de la lluvia(16).

En relación al grupo etario (ver tabla 2), la presencia de anticuerpos IgG contra el dengue fue más frecuente en habitantes con edades comprendidas entre $10 \mathrm{y}$ 20 años (8,7 \% OR: 0,16; PR: 0,14; IC95 \%: 0,08-0,21). Además, no se encontró asociación significativa entre la edad y la seroprevalencia de dengue ( $p>0,05)$. Diversos estudios han reportado grupos etarios muy variables sin encontrarse un patrón definido ${ }^{(17-19)}$. Al respecto, Castañeda et al. ${ }^{(19)}$ manifestaron que el dengue puede afectar a cualquier grupo etario; sin embargo, son los jóvenes y adultos jóvenes los que pueden ser especialmente perjudicados, debido a las brechas educativas y a falta de oportunidades para su desarrollo, lo que conlleva al desconocimiento sobre la enfermedad y las formas de prevenirla.

En la tabla 2, se evidenció que el 9,3\% de estudiantes (OR: 0,18; PR: 0,16; IC95 \%: 0,09-0,23) y el 5,8 \% de amas de casa (OR: 0,31; PR: 0,24; IC95 \%: 0,11-0,37) fueron seropositivos al dengue. Resultados similares fueron los obtenidos por Niño y Yong ${ }^{(11)}$ y Chilón ${ }^{(20)}$, quienes determinaron los factores asociados al dengue en los distritos de Tumán (Chiclayo) y Tanta Arica (Contumaza), respectivamente, encontrando que las amas de casa fueron uno de los grupos más afectado por la enfermedad.

Las amas de casa se encontrarían más expuestas a las picaduras del $A$. aegypti, debido a las actividades que realizan, permaneciendo en el hogar por más largos periodos de tiempo, en comparación con los pobladores que tienen otras ocupaciones. Así también, Pereyra et al. (1) reportaron que el grupo ocupacional más frecuentemente afectado por el dengue fueron las amas de casa $(18,1 \%)$, atribuyendo este resultado al estilo de vida que poseen. En relación a los estudiantes, se considera que los centros educativos podrían constituir zonas de riesgo donde se multiplica el vector, siendo necesario impulsar la difusión de las medidas de prevención y promoción para su control.

El centro poblado Fila Alta no cuenta con abastecimiento de agua durante las 24 horas del día, por lo que sus pobladores comúnmente almacenan el agua en diferentes depósitos y por periodos de tiempo prolongado. En esta investigación se encontró que el $72,1 \%$ de los pobladores recolectaban agua para su consumo en tanques, encontrándose anticuerpos lgG en el 15,7 \% de estos pobladores (OR: 0,28; PR: 0,22; IC95 \%: $0,15-0,29)$ (ver tabla 2). Según Chilon ${ }^{(20)}$, la presencia de los depósitos para la recolección de agua de consumo dentro de las viviendas con irregular abastecimiento de este servicio favorece la diseminación del vector e incrementa el riesgo de infección (86,6 \%).

Se conoce que el vector del dengue es capaz de adaptarse fácilmente a diferentes ambientes, reproduciéndose no solo en la superficie del agua limpia almacenada en distintos recipientes, sino también en agua estancada o sucia. Al respecto, el servicio departamental de La Paz, Bolivia (SEDES) ha identificado larvas del mosquito en aguas servidas ${ }^{(21)}$. Asimismo, Mariné et al. ${ }^{(22)}$ compararon los datos de la vigilancia ambiental de dos grupos vecinales en el municipio Cotorro, La Habana, con la finalidad de prevenir el dengue, observando 
que dicha población mantenía en muchas ocasiones los recipientes descubiertos o cubiertos solo con un mantel, debido a la practicidad que representa, considerando el tipo y tamaño de los depósitos y la frecuencia con que estos eran usados. Similar situación fue la observada en los residentes de Fila Alta, lo que puede favorecer la multiplicación del mosquito vector. Sumado a ello, las temperaturas que oscilan entre 26 y $28^{\circ} \mathrm{C}$ incrementan la cinética del desarrollo y supervivencia del A. aegypti, en cualquiera de sus etapas ${ }^{(23)}$.

Referente al material de las viviendas (ver tabla 2), se evidenció que el $8,7 \%$ de pobladores seropositivos al DENV habitaba en viviendas construidas a base de adobe (OR: 0,94; PR: 0,48; IC95 \%: 0,30-0,66), seguido de un 8,1 \% a base de ladrillos (OR: 0,18; PR: 0,15; IC95 \%: 0,08-0,22). Los resultados obtenidos se asemejan a los recopilados por Nagua ${ }^{(24)}$, en cuyo estudio encontró que un 11,0\% de pobladores de Machala (Venezuela) tenían viviendas de adobe. El adobe es un material de construcción temporal muy utilizado en las viviendas del departamento de Cajamarca. Sobre su superficie usualmente aparecen grietas y el mosquito que transmite el dengue suele albergarse en ellas. Por lo que, para Agüero-Vega y Pando ${ }^{(25)}$, el material presente en las paredes de las viviendas es un factor asociado a la seropositividad de dengue, coincidiendo con lo establecido en esta investigación $(p<0,05)$.

En base a los resultados obtenidos, se concluyó que la seroprevalencia de la infección por dengue fue alta (19,2\%) y estuvo asociada significativamente a la ocupación de los pobladores y el material utilizado para la construcción de sus viviendas. Ante ello, es importante resaltar que para el control del vector y la consecución de resultados favorables en la lucha contra el dengue es primordial además de un diagnóstico oportuno de la enfermedad, impulsar la educación y participación activa de la población.

\section{REFERENCIAS}

1. Pereyra Y, Samudio M, Ojeda A, Cabello Á. Seroprevalencia de la infección por dengue en el distrito de Chaco Paraguayo. Estudio Poblacional. Rev Chil Infectol. [Internet] 2015 Dic; 32(6): 618-27 [Consultado 2020 Dic 20]. Disponible en: http://dx.doi.org/10.4067/S071610182015000700002

2. Dengue y dengue grave [Internet] Ginebra: Organización Mundial de la Salud; 2020 [Consultado 2021 Feb 20] Disponible en: https://www.who.int/es/news-room/ fact-sheets/detail/dengue-and-severe-dengue

3. Frantchez V, Fornelli R, Sartori G, Arteta Z, Cabrera S, Sosa $L$, et al. Dengue en adultos: diagnóstico, tratamiento y abordaje de situaciones especiales. Rev. Méd. Urug. [Internet] 2016 Abr; 32(1): 43-51 [Consultado 2021 Feb 18] Disponible en: http://www.scielo.edu.uy/scielo.php?s-
cript=sci_arttext\&pid=S1688-03902016000100006\&ln$\mathrm{g}=\mathrm{es}$

4. World Health Organization (WHO). Global strategy for dengue prevention and control 2012-2020. Geneva: WHO; 2012.

5. Dengue [Internet]. Perú: Organización mundial de la salud; 2020 [Consultado 2021 Feb 20] Disponible en: https://www.paho.org/per/index.php?option=com content\&view=article\&id=4079:dengue\&ltemid=0\#: :text=En\%20el\%20Per\%C3\%BA\%2C\%20durante $\% 20$ el,fue $\% 20$ de $\% 200 \% 2$ C12\%25

6. Reyes A, Guzmán MG, Ramírez R, Hernández C, García $\mathrm{G}$, Torres DM, et al. Impacto económico del dengue en pacientes y familiares. Rev Cuba Med Tropical [Internet] 2019 Jun; 71(3):1-19 [Consultado 2021 Mar 18 Mar] Disponible en: http://revmedtropical.sld.cu/index.php/medtropical/article/view/419

7. Luna H, Gómez G, Cando Wilson C. Factores epidemiológicos asociados a dengue en pacientes adultos. Revista Científica Mundo de la Investigación y el Conocimiento [Internet] 2018 Jun; 2 (2): 700-709 [Consultado $2021 \mathrm{Mar}$ 18] Disponible en: http://dx.doi.org/10.26820/recimundo/2.(2).2018.700-709

8. Villa LP. Factores de riesgo asociados al dengue. Rev. enferm. vanguard. [Internet] 2017 Ene; 5(1): 1-2 [Consultado 2021 Mar 18]. Disponible en: https://doi. org/10.35563/revan.v5i1.221

9. Arredondo-García JL, Méndez-Herrera A, Medina-Cortina $H$. Arbovirus en Latinoamérica. Acta pediátrica de México [Internet] 2016 Mar; 37(2): 111-131 [Consultado 2021 Feb] Disponible en: http://www.scielo.org.mx/scielo.php?script=sci_arttext\&pid=S018623912016000200111\&ln$\mathrm{g}=\mathrm{es} \& \mathrm{t} \operatorname{lng}=\mathrm{es}$

10. Blacksell SD, Newton PN, Bell D, Kelley J, Mammen MP, Vaughn DW, et al. The comparative accuracy of $8 \mathrm{com}$ mercial rapid immunochromatographic assays for the diagnosis of acute dengue virus infection. Clin Infect Dis. [Internet] 2006 Abr; 42(8):1127-34 [Consultado 2021 Feb 20] Disponible en: https://doi.org/10.1086/501358

11. Niño S, Yong A. Conocimientos y prácticas en prevención de dengue post fenómeno del niño costero. Tumán - Chiclayo, 2018 [Tesis de grado]. Lambayeque: Universidad nacional Pedro Ruiz Gallo; 2018.

12. Vircell Microbiologists. Dengue ELISA IgG - Producto para diagnóstico in vitro [Internet] [Consultado 2019 Sep 20] Disponible en: https://www.vircell.com/media/ INSERTS/DENGUE\%20ELISA\%20lgG_G1018_ES.pdf

13. Velandia ML, Castellanos JE. Virus del dengue: estructura y ciclo viral. Infectio. [Internet] 2011 Feb; 15: 33-43 [Consultado 2021 Mar 15] Disponible en: http://dx.doi. org/10.1016/S0123-9392(11)70074-1

14. Neira M. Factores de riesgo asociados a infección por dengue en familias del centro poblado Río Seco, El Porvenir, Trujillo, La Libertad, 2017 [Tesis de licenciatura en Enfermería] La Libertad: Universidad Cesar Vallejo; 2018.

15. Molineros LF, Pinzón Gómez EM, Rengifo García NE, Daza CF, Hernández-Carrillo M, Ortiz ME, et al. Seroprevalencia de dengue en municipios con transmisión hiperendémica y mesoendémica, Valle del Cauca, Colombia. Rev Cubana Salud Pública[Internet] 2020 Abr; 46(2): 1-20 [Consultado 2021 Mar 2] Disponible en: http:// www.revsaludpublica.sld.cu/index.php/spu/article/ view/1256 
16. Cabezas C, Fiestas V, García-Mendoza M, Palomino M, Mamani E, Donaires F. Dengue en el Perú: a un cuarto de siglo de su reemergencia. Rev Peru Med Exp Salud Publica. 2015; 32(1):146-56.

17. Gutiérrez C, Montenegro J. Conocimiento sobre dengue en una región endémica de Perú. Estudio de base poblacional. Acta Médica Peruana [Internet] 2017 Oct; 34(4): 283-288 [Consultado 2021 Mar 2] Disponible en: http://www.scielo.org.pe/scielo.php?script=sci_arttext\&pid=S1728-59172017000400005\&Ing=es

18. Caraballo A, Chica I, Ocampo M. Comportamiento epidemiológico del Dengue, Chikungunya y Zika. Córdoba-Colombia. Rev. Colombiana Salud Libre [Internet] 2017 Dic; 12(2): 7-14 [Consultado 2021 Mar 2] Disponible en: https://doi.org/10.18041/1900-7841/rcslibre.2017v12n2.1725

19. Castañeda-Porras O, Segura O, Garon-Lara E, Manosalva-Sánchez C. Conocimientos, actitudes y prácticas frente al control del vector Aedes aegypti, Villanueva. Casanare, Colombia. Rev. Médica de Risaralda[Internet] 2017 Dic; 23(2): 14-22 [Consultado 2021 Mar 2] Disponible en: http://www.scielo.org.co/scielo.php?script=sci_arttext\&pid=S0122-06672017000200003\&lng=en

20. Chilon S. Factores de riesgo asociados al brote epidémico de Dengue en el Centro Poblado Menor El Salitre - Cajamarca 2017 [Tesis de maestría en Ciencias] Cajamarca: Universidad Nacional de Cajamarca; 2018.
21. Céspedes LM, Mendoza G, Díez M. Caso inusual de dengue. Rev. bol. ped [Internet] 2012 Abr; 51(1): 15-18 [Consultado 2021 Mar 10] Disponible en: http://www. scielo.org.bo/scielo.php?script=sci_arttext\&pid=S102406752012000100004\&lng=es

22. Mariné $M$, García $M$, Torres $Y$, Vásquez $M$. Comparación de datos de la vigilancia ambiental y de grupos vecinales para prevenir el dengue. Rev Cubana Hig. Epidemiol. [Internet] 2007 Abr; 45(1): 28-59 [Consultado $2021 \mathrm{Mar}$ 10] Disponible en: http://scielo.sld.cu/scielo.php?scrip$\mathrm{t}=\mathrm{sci}$ _arttext\&pid=S1561-30032007000100008\&lng=es

23. Marinho RA, Beserra EB, Bezerra-Gusmão MA, Porto V de S, Olinda RA, dos Santos CAC. Effects of temperature on the life cycle, expansion, and dispersion of Aedes aegypti (Diptera: Culicidae) in three cities in Paraiba, Brazil. JVector Ecol. [Internet] 2016 Jun; 41(1): 1-10 [Consultado 2021 Mar 15] Disponible en: https://doi.org/10.1111 / jvec.12187

24. Nagua C. Dengue en personas de 20-30 años de edad que acuden al sub centro de salud Venezuela del Cantón Machala del mes de enero a julio del año 2012 [Tesis de pregrado en Enfermería] Ecuador: Universidad Técnica de Machala; 2014.

25. Agüero-Vega A, Ramos-Pando W. Asociación entre los casos de dengue con las características de la vivienda y conocimiento sobre la enfermedad. Rev Peru Investig Salud [Internet] 2018 Dic; 2(2): 24-29 [Consultado $2021 \mathrm{Mar}$ 15] Disponible en: https://doi.org/10.35839/repis.2.2.221 